\title{
EDITORIAL
}

\section{Lighter than air: heliox breathing improves exercise tolerance in COPD}

\author{
P. Palange
}

D yspnoea and reduced exercise tolerance are the hallmarks of chronic obstructive pulmonary disease (COPD). In the advanced phases of the disease the ability to exercise is mainly limited by the combination of a reduced ventilatory capacity, increased ventilatory requirement and lung dynamic hyperinflation. Importantly, the aforementioned respiratory mehanical abnormalities are well recognised causes of exertional dyspnoea in COPD [1]. However, in a substantial number of patients a heightened perception of leg effort may reduce tolerance to cycle-ergometer exercise, while dyspnoea appears to be the main symptom limiting the ability to walk [2]. Regardless of this, there would be little question that, in patients with severe COPD and particularly in those subjects with predominant emphysema, maximal exercise tolerance is markedly reduced because of ventilatory limitation, dynamic hyperinflation and dyspnoea sensation.

Figure 1 illustrates the lung mechanical abnormalities that cause dyspnoea and exercise intolerance in COPD. Airflow obstruction could be the result of intrinsic airways narrowing, as observed in chronic bronchitis, or may be the result of airways collapse due to loss of lung elastic recoil, as observed in emphysema. Importantly, both these anatomical alterations cause a reduction in expiratory flow reserve. When this occurs, breathing is usually accommodated to a higher lung volume, to prevent the unpleasant sensation arising from the central airways and to permit adequate ventilation. As a result, dynamic lung hyperinflation develops due to well-refined actions of various neural and mechanical mechanisms, presumably to achieve the best compromise between the minimum amount of expiratory flow limitation and the least load for the inspiratory muscles. Breathing under conditions of expiratory flow limitation is associated with an increased work of breathing (WOB) and could lead to two basic physiological consequences: 1) airways undergoing mechanical collapse trigger neural signals elaborated at the cortical level, i.e. dyspnoea sensation; and 2) the high intrathoracic and abdominal pressures resulting from an elevated expiratory effort may limit venous return to the right heart. A reduction in cardiac output and oxygen delivery to the exercising muscles has been postulated as a pathopysiological

CORRESPONDENCE: P. Palange, Dept of Clinical Medicine, University of Roma "La Sapienza", v.le Università 37, 00185 Rome, Italy. E-mail: paolo.palange@uniroma1.it

Received: Jan 03 2010; Accepted: Jan 052010

PROVENANCE: Submitted article, peer reviewed. mechanism affecting exercise tolerance in severe COPD patients at the maximal level of exercise.

Different strategies have been utilised to improve lung mechanics, dyspnoea and exercise tolerance in COPD, such as decreasing bronchial tone (by the use of bronchodilators) and/or reducing the amount of ventilation needed at a given work rate (by the use of exercise based rehabilitation programmes). Both strategies, alone or in combination, appear to be capable of improving exercise tolerance and dyspnoea on exertion in COPD $[3,4]$.

Studies have been also carried out to demonstrate the beneficial effect of "ergogenic aids" on exercise, such as oxygen supplementation [5]. The effects of breathing lowdensity gas mixtures (i.e heliox or helium+oxygen) during exercise have only been tested recently: the results of eight important studies published in this field have been reviewed by HunT et al. [6] in the current issue of the European Respiratory Review. In their study, Hunt et al. [6] clearly illustrate the rationale beyond the use of heliox during exercise in COPD and adequately summarise the relevant results of these research articles. Importantly, as for the results of their review analysis, HunT et al. [6] concluded that: "Overall, the eight studies included in this review presented a case for high level, low risk of bias evidence to support the effectiveness of heliox in improving the intensity and endurance of exercise when compared to room air for people with COPD.". In contrast, the review by HUNT et al. [6] failed to demonstrate a significant reduction in exertional dyspnoea induced by heliox breathing. It should be noted, however, that dyspnoea was not the primary outcome in these studies and that differences in the studied patient population and exercise protocols utilised (i.e. maximal versus constant work rate and cycling versus walking), may have contributed to this result.

\section{HOW DOES HELIOX WORK?}

It is important to remember that maximum flow in the airways is determined not only by their size and compliance at the choke point but also by physical properties of the inhaled gases. During heliox breathing, the density of which is approximately one third of air, flow is expected to increase as a result of decreased turbulence within the large airways. Helium based mixtures are mainly utilised in deep diving, e.g. > 6ATM (5.92 bar): in fact, the high environmental pressure reached during diving induces airflow limitation and increases the WOB [7]. In this environmental condition heliox acts by diminishing the airflow resistance and by decreasing turbulences. It is important to note that flow limitation that 


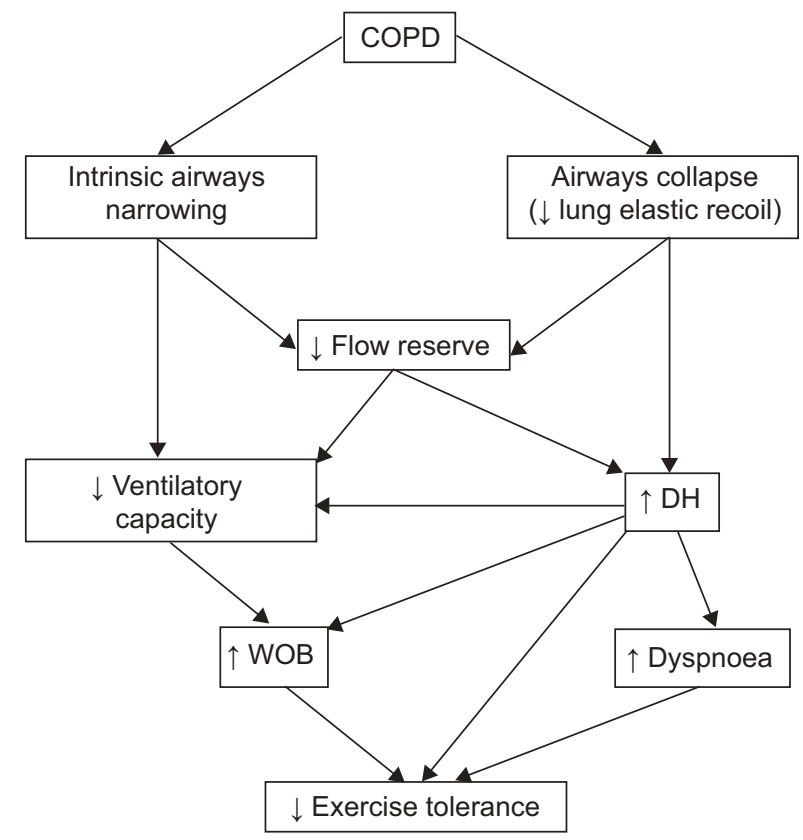

FIGURE 1. Lung mechanical abnormalities causing exercise intolerance in chronic obstructive pulmonary disease (COPD). DH: dynamic hyperinflation; WOB: work of breathing. Refer to the text for comments.

occurs when breathing air in high-pressure environments represents a condition similar to that experienced by severe COPD patients during high-intensity bouts of exercise.

\section{WHY DOES HELIOX IMPROVE EXERCISE TOLERANCE IN COPD?}

In normal individuals heliox breathing induces sustained exercise hyperventilation and hypocapnia [8,9] because this mixture is capable of accommodating greater maximum flow and ventilation and allows tidal volume to increase at the expense of a reduced functional residual capacity. In patients with severe airways obstruction, turbulent flow frequently occurs, even at low breathing rates [10]; total airway resistance (Raw) is very high and leads to airflow limitation and increased work of breathing. The increase in Raw is mainly due to narrowing of the airways due to inflammatory infiltration or due to reduced lung elastic recoil. It has been shown that the increase in Raw mainly occurs in peripheral airways $(<3 \mathrm{~mm}$ diameter), although a significant increase in Raw could also be observed in the major intrathoracic conducting airways [11]. The eight studies revised by HuNT et al. [6] were mainly conducted in moderate-to-severe COPD patients. The more favourable and convincing effect of heliox breathing was the significant improvement in exercise endurance time; this was mostly due to an improvement in ventilatory capacity and lung mechanics (e.g. reduced lung dynamic hyperinflation and WOB). Recently, CHIAPPA et al. [12] confirmed the positive effect of heliox breathing on lung pulmonary mechanics and, ultimately, on exercise tolerance. In addition, CHIAPPA et al. [12] were able to demonstrate a favourable effect of heliox on peripheral muscle oxygenation. It is important to point out that the studies which were able to demonstrate a significant improvement in exercise tolerance with heliox all utilised a high-intensity constant work-rate (CWR) "endurance" protocol [13-15]. This "exercise strategy" has been also successful in showing the positive effects of other therapeutic interventions, such as bronchodilators, oxygen supplementation and exercisebased pulmonary rehabilitation. Nowadays, the high-intensity CWR endurance protocol appears to have a greater sensitivity compared with the incremental exercise test in evaluating the effects of therapeutic interventions [16]. This is probably due to the fact that even small positive changes in lung mechanics can translate into significant positive changes in endurance tolerance. In a recent study on the effect of heliox in COPD it was speculated that: "small increment in FEV1 (e.g. $0.16 \mathrm{~L}$ ) induced by heliox at rest can be roughly translated in an increase in maximal ventilation of $5.6 \mathrm{~L} \cdot \mathrm{min}^{-1}$ that, in a COPD patient with $1.3 \mathrm{~L}$ of $\mathrm{FEV} 1$, predicts a $12 \%$ increase in maximal ventilatory capacity." [13].

As discussed previously, CWR has been found to be useful in evaluating the effect of an intervention. If a given work rate can be sustained for a longer period after an intervention this is taken as evidence of improved exercise tolerance. Furthermore, if the increase in exercise tolerance is associated with amelioration in physiological response variables, this constitutes an effort independent of evidence of improvement in exercise tolerance. It should be clarified, however, that the selection of the work rate to be used for the CWR must be individualised to the patient's maximal exercise tolerance [17]. In fact, if the work rate selected is too high the CWR test will be tolerated for only a very short duration of time and improvements in the physiological ability to exercise will result in very small or no improvements in exercise time. If the work rate is too low then the CWR exercise will last indefinitely and will not be a helpful discriminant. It has been suggested that exercise durations in the range of $4-7 \mathrm{~min}$ are desirable baselines for interventional studies. Importantly, it has been demonstrated that in patients with moderate-to-severe COPD, choosing a work rate of $80-85 \%$ of the peak work rate measured at the incremental test yielded the best chance of achieving a test duration of 4-7 $\mathrm{min}$ [18].

In conclusion, the good news is that heliox breathing increases exercise "endurance" tolerance in severe COPD patients. What is not yet understood is which patients could benefit the most from heliox breathing, and if these patients could be identified by the use of resting lung function measurements. Further studies are also needed to verify the utility of heliox as an ergogenic aid to training in pulmonary rehabilitation. Because of the elevated cost of helium, it will be also interesting to see if helium sparing devices will be developed in the near future.

\section{STATEMENT OF INTEREST}

None declared.

\section{REFERENCES}

1 O'Donnell DE. Hyperinflation, dyspnea, and exercise intolerance in chronic obstructive pulmonary disease. Proc Am Thorac Soc 2006; 3: 80-184.

2 Palange $\mathrm{P}$, Forte $\mathrm{S}$, Onorati $\mathrm{P}$, et al. Ventilatory and metabolic adaptations to walking and cycling in COPD. J Appl Physiol 2000; 88: $1715-1720$. 
3 O'Donnell DE, Flüge T, Gerken F, et al. Effects of tiotropium on lung hyperinflation, dyspnoea and exercise tolerance in COPD. Eur Respir J 2004; 23: 832-840.

4 Casaburi R, Kukafka D, Cooper CB, et al. Improvement in exercise tolerance with the combination of tiotropium and pulmonary rehabilitation in patients with COPD. Chest 2005; 127: 809-817.

5 O'Donnell DE, D'Arsigny C, Webb KA. Effects of hyperoxia on ventilatory limitation during exercise in advanced chronic obstructive pulmonary disease. Am J Respir Crit Care Med 2001; 163: 892-898.

6 Hunt T, Williams MT, Frith P, et al. Heliox, dyspnoea and exercise in COPD. Eur Respir Rev 2010; 19: 30-38.

7 Van Liew HD. Mechanical and physical factors in lung function during work in dense environments. Undersea Biomed Res 1983; 10: 255-264.

8 Babb TG. Ventilatory response to exercise in subjects breathing $\mathrm{CO}_{2}$ or $\mathrm{HeO}_{2}$. J Appl Physiol 1997; 82: 746-754.

9 Ward SA, Whipp BJ, Poon CS. Density-dependent airflow and ventilatory control during exercise. Respir Physiol 1982; 49: 267-277.

10 Otis A, Bembower W. Effect of gas density on resistance to respiratory gas flow in man. J Appl Physiol 1949; 2: 300-306.

11 Yanai M, Sekizawa K, Ohrui T, et al. Site of airway obstruction in pulmonary disease: direct measurement of intrabronchial pressure. J Appl Physiol 1992; 72: 1016-1023.
12 Chiappa GR, Queiroga F Jr, Meda E, et al. Heliox improves oxygen delivery and utilisation during dynamic exercise in patients with chronic obstructive pulmonary disease. Am J Respir Crit Care Med 2009; 179: 1004-1010.

13 Palange $P$, Valli G, Onorati $P$, et al. Effect of heliox on lung dynamic hyperinflation, dyspnea, and exercise endurance capacity in COPD patients. J Appl Physiol 2004; 97: 1637-1642.

14 Eves ND, Petersen SR, Haykowsky MJ, et al. Helium-hyperoxia, exercise, and respiratory mechanics in chronic obstructive pulmonary disease. Am J Respir Crit Care Med 2006; 174: 763-771.

15 Laude EA, Duffy NC, Baveystock C, et al. The effect of helium and oxygen on exercise performance in chronic obstructive pulmonary disease: a randomized crossover trial. Am J Respir Crit Care Med 2006; 173: 865-870.

16 Palange $\mathrm{P}$, Ward SA, Carlsen $\mathrm{K}-\mathrm{H}$, et al. Recommendations on the use of exercise testing in clinical practice. Eur Respir J 2007; 29: 185-209.

17 Whipp BJ, Ward SA. Quantifying intervention-related improvements in exercise tolerance. Eur Respir J 2009; 33: 1254-1260.

18 Casaburi R. Factors determining constant work rate exercise tolerance in COPD and their role in dictating the minimal clinically important difference in response to interventions. COPD 2005; 2: 131-136. 